\title{
Local gravity from Lunar Prospector tracking data: Results for Mare Serenitatis
}

\author{
S. Goossens ${ }^{1,2}$, P. N. A. M. Visser ${ }^{1}$, K. Heki ${ }^{3}$, and B. A. C. Ambrosius ${ }^{1}$ \\ ${ }^{1}$ Department of Earth Observation and Space Systems, section Astrodynamics and Satellite Systems, Faculty of Aerospace Engineering, \\ Delft University of Technology, Kluyverweg 1, 2629 HS Delft, The Netherlands \\ ${ }^{2}$ National Astronomical Observatory Japan, Mizusawa Astrogeodynamics Observatory, 2-12 Hoshigaoka, Mizusawa-city, \\ Iwate 023-0861, Japan \\ ${ }^{3}$ Division of Earth and Planetary Sciences, Hokkaido University, Kita-ku, N10 W8, Sapporo 060-0810, Japan
}

(Received May 25, 2005; Revised July 25, 2005; Accepted July 28, 2005)

\begin{abstract}
High-resolution gravity anomalies on the surface of the Moon are determined from Lunar Prospector tracking data residuals. By means of a benchmark test the recovery method is validated with respect to the orbit determination and gravity field recovery strategy. Tracking data for the entire extended mission of Lunar Prospector, during which the satellite flew at an average altitude of $30 \mathrm{~km}$ above the lunar surface, have been completely and independently processed and orbits have been determined. Using tracking data residuals from these precise orbits, adjustments to the a priori gravity field model have been created for Mare Serenitatis. The results for Mare Serenitatis with the local recovery are comparable to global recovery results, yet faster and more efficient with a possibility to increase the resolution.
\end{abstract}

Key words: Lunar gravity, local and regional gravity, Lunar Prospector, Mare Serenitatis.

\section{Introduction}

Until this date, the lunar gravity field has mainly been expressed by a global spherical harmonical representation, despite the lack of tracking data over the far side of the Moon. Such a representation requires regularisation such as taking a priori information into account in order to have a stable and meaningful solution. Floberghagen (2002) has shown that the choice of this a priori information greatly affects the complete final solution, for the far side as well as for the near side. To extract the wealth of information about the near side present in tracking data of the Lunar Prospector spacecraft and to overcome problems inherent to the use of global spherical harmonic basis functions, regional representations become of interest. They can be used to derive accurate and high-resolution local and regional (depending on the size of the area) gravity anomaly maps of the near side, which can help to understand the structure and evolution of the Moon, and consequently the Earth-Moon system, as they provide the boundary conditions for internal processes and structure. Such a regional method also has the advantage that it is fast and efficient.

This paper presents a high-resolution local gravity anomaly solution for Mare Serenitatis, one of the large mascons on the near side. Such a high-resolution solution can for instance be used in combination with topography to study the characteristics of mare fill and the sub-surface structure, as was done by Watters and Konopliv (2001). The solution presented here has been obtained by processing Lu-

Copyright (c) The Society of Geomagnetism and Earth, Planetary and Space Sciences (SGEPSS); The Seismological Society of Japan; The Volcanological Society of Japan; The Geodetic Society of Japan; The Japanese Society for Planetary Sciences; TERRAPUB nar Prospector tracking data. These data have been processed using the GEODYN II software package (Rowlands et al., 1995). Residuals from orbit determination done with this software package have been used to determine adjustments in terms of gravity anomalies to the reference gravity field model, which was LP150Q, the latest Lunar Prospector spherical harmonic solution and a result of a full-rank inversion in one step (as opposed to its predecessor LP165P (Konopliv et al., 2001)). A complete and independent reprocessing of Lunar Prospector tracking data using stateof-the-art reference models allows for a comprehensive and accurate determination of local gravity fields on the Moon.

\section{Method and Benchmark Test}

The recovery method uses a linear variational approach, and is an extension of the method presented by Vonbun et al. (1980). The method and its sensitivity with respect to various error sources are discussed in great detail in Goossens et al. (2005) and Goossens (2005). A Doppler residual $\Delta \dot{\rho}$ can be thought to be caused by local gravity anomalies. In a linear variational form, by using a first order Taylor expansion, the dependency of the residual on the vector of anomalies $\Delta \vec{g}$ can be expressed as:

$$
\Delta \dot{\rho}=\frac{\partial \dot{\rho}}{\partial \vec{x}_{\text {sat }}} \frac{\partial \vec{x}_{\text {sat }}}{\partial \Delta \vec{g}} \Delta \vec{g}
$$

where $\vec{x}_{\text {sat }}$ is the 6 parameter satellite state vector consisting of position and velocity and $\partial \dot{\rho} / \partial \vec{x}_{\text {sat }}$ is a geometrical partial. The vector $\Delta \vec{g}$ is a vector containing the gravity anomalies for each grid point on a reference sphere with a radius of $1738 \mathrm{~km}$. The anomalies are expressed as the magnitude of the acceleration vector and are related to the 


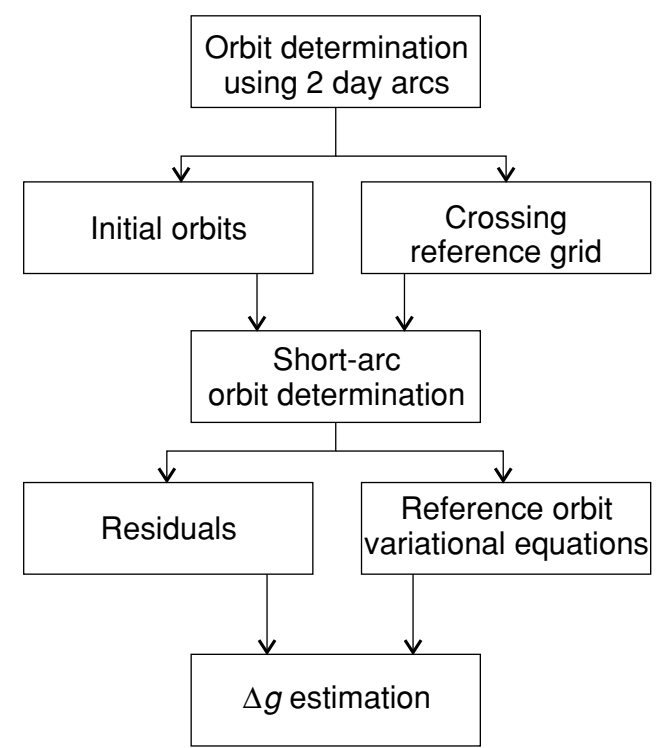

Fig. 1. Flow chart local gravity field recovery.

disturbing potential $T$ in the spherical approximation by $\Delta g=-\partial T / \partial r-2 T / r$, where $r$ is the radius, see e.g. Heiskanen and Moritz (1984). The partial $\partial \vec{x}_{\text {sat }} / \partial \Delta \vec{g}$ describes the sensitivity of the components of the state vector with respect to the separate anomalies per grid cell. This sensitivity matrix can be determined by integration of the variational equations. Previous local and regional gravity field determination efforts (such as the recent result for the Moon by Sugano and Heki (2004)), often used line-of-sight accelerations, obtained by numerically differentiating the Doppler residuals. In this paper, the Doppler residuals are used directly, in order to avoid the delicate matters of numerical differentiation, which can introduce errors on the data, as was noted as well by Barriot et al. (1997).

This method and the data processing strategy have been validated by means of a benchmark simulation using the GEODYN II software. In this test case, the processing of the actual Lunar Prospector data, and the subsequent local gravity recovery, have been simulated. The steps that are taken in the recovery process are schematically drawn in

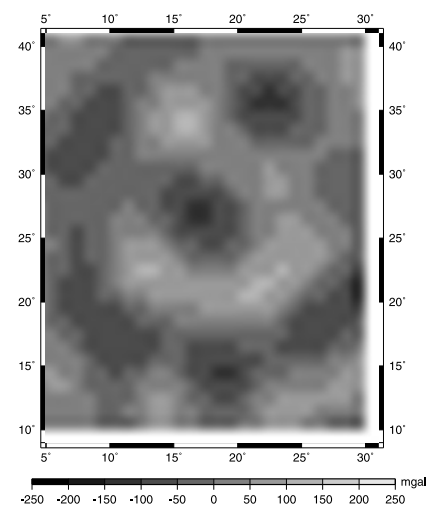

Input $\Delta \vec{g}, \mathrm{LP} 150 \mathrm{Q}, l=21-50$

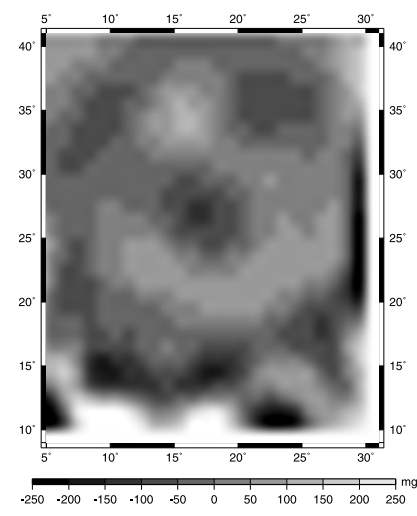

$M$ fixed

Fig. 1. A truth orbit has been generated using the LP150Q lunar gravity field model up to degree $l=50$, at an average satellite altitude of $100 \mathrm{~km}$. This orbit is equivalent to the " 2 day arc orbit" mentioned in Fig. 1. Orbits obtained from spans of two days (called arc) will be used as starting point when real Lunar Prospector data are processed. This truth orbit will pass over Mare Serenitatis, which is located in the area $\lambda=5^{\circ}-30^{\circ} \mathrm{E}$ and $\phi=10^{\circ}-40^{\circ} \mathrm{N}$. This will also be the recovery area, consisting of 806 anomaly blocks of $1^{\circ} \times 1^{\circ}$, where the given coordinates denote the location of the central points of the anomaly blocks. This truth orbit thus determines when the satellite actually crosses the area under consideration, and it also delivers the initial state vectors for the next step.

Simulated data will consist of two-way range and Doppler data. Stations used are DSN stations 24, 42 and 61 , with coordinates as published by DSN (Thornton and Border, 2000). These simulated data will be used in a reduction run, which uses the same models, except that the gravity field model is now used up to degree $l=20$. That means that the discrepancy between both runs represented by $l=21-50$ should be present in the final recovered anomalies. Orbits will be determined in this reduction run using a short-arc approach: one arc is defined as the time it takes the satellite to travel over the recovery area on the lunar surface. With the chosen area on the Moon, this results in arcs of about ten minutes. As shown in Fig. 1, the starting point (in time as well as in terms of the initial state vector) for these short arcs is taken from the truth orbit. Performing orbit determination with such short arcs requires extra efforts in order to have convergence of the orbit determination process. In Goossens et al. (2005) and Goossens (2005) state vector estimation in short-arc orbit determination has been investigated, and by means of eigenvalue analysis of the part of the normal matrix for the state vector adjustment it was found that only certain combinations of the adjustment are observable rather than the full 6 element adjustment. In total, 3 or 4 eigenvalues were found to be numerically close to zero, leaving only two parameters that are actually observable. Therefore, it is chosen here to fix 3 or 4 selected Kepler elements in the orbit determination. The Kepler elements that are kept fixed here will be the ec-

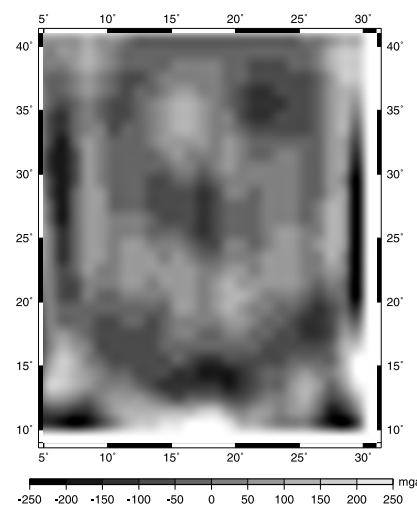

$M$ not fixed

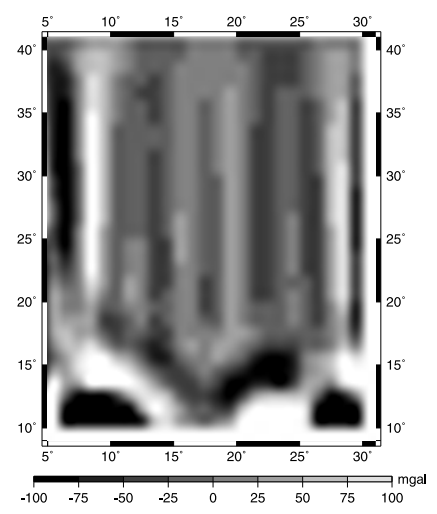

Differences $M$ fixed or not

Fig. 2. Input and recovered anomalies in the benchmark test for the Mare Serenitatis area. Input anomalies coming from LP150Q, $l=21-50$, have an $\mathrm{rms}$ of $58.34 \mathrm{mGal}$; recovered anomalies with $M$ fixed have an rms of $55.54 \mathrm{mGal}$, and anomalies where $M$ is not fixed have an rms of $57.09 \mathrm{mGal}$. These values are for the central part of the anomalies only $\left(\lambda=10^{\circ}-25^{\circ} \mathrm{E}, \phi=15^{\circ}-35^{\circ} \mathrm{N}\right)$. Tikhonov regularisation has been used to obtain the solutions. 
centricity $e$, the right-ascending node $\Omega$ and the argument of pericenter $\omega$. The right-ascending node is chosen since it will fix the orientation of the orbital plane. To maintain some flexibility, the inclination, which also defines part of the orientation of the orbital plane, is not chosen here. The argument of pericenter is chosen together with the eccentricity since they are related for near-circular orbits. The argument of pericenter is also closely related to the mean anomaly, especially for such short arcs. The mean anomaly $M$ itself can also remain fixed in order to compensate for the fourth small eigenvalue; the effect of this is investigated. Results from these short-arc orbit determinations are a set of residuals and an orbit. These residuals, which carry the information about the discrepancy in force models between the truth orbit and the results from the reduction run, are the data points in the local gravity field recovery, and these orbits are used as the reference orbits along which the variational equations are integrated, cf. Fig. 1. This then yields all the elements for Eq. (1), written in the standard form $\vec{y}=\mathbf{A} \vec{x}$, to be solved. Here, $\vec{x}$ is the vector with the anomalies (not to be confused with the state vector $\vec{x}_{\text {sat }}$ ), $\vec{y}$ the vector with the data points, and $\mathbf{A}$ the design matrix, the entries of which consist of the partials of Eq. (1).

Results for the benchmark simulation are shown in Fig. 2. This figure shows both the input anomalies (those from LP150Q for $l=21-50$ ) as well as recovered anomalies. The recovered anomalies have been obtained by either estimating the mean anomaly $M$ in the short-arc orbit determination or not. Both recovered anomalies use Tikhonov regularisation, where the solution $\hat{x}_{\alpha}$ is obtained by $\hat{x}_{\alpha}=\left(\mathbf{A}^{T} \mathbf{W A}+\alpha \mathbf{I}\right)^{-1} \mathbf{A}^{T} \mathbf{W} \vec{y}$, where $\mathbf{W}$ is a diagonal weight matrix for the data, using $1 \mathrm{~m}$ for range data and 1.0 $\mathrm{mm} / \mathrm{s}$ for Doppler data, and $\mathbf{I}$ is the identity matrix. The regularisation parameter $\alpha$ was chosen to be $10^{+7}$, the optimal value obtained from simulations. The results show that the input anomalies can be recovered. The rms of differences for the central part of the solution (which covers $\lambda=10^{\circ}-$ $25^{\circ} \mathrm{E}$ and $\phi=15^{\circ}-35^{\circ} \mathrm{N}$, and which is taken here because boundary effects are intrinsic to local and regional recovery methods; for an assessment of this effect, see Goossens et al. (2005) and Goossens (2005)) between input and recovered anomalies is $15.69 \mathrm{mGal}$ when $M$ is fixed and 27.06 $\mathrm{mGal}$ when $M$ is not fixed. As can also be seen from Fig. 2, the solution with $M$ fixed resembles the input signal best; the solution with $M$ free shows orbital patterns. The differences between both solutions, with either fixing or not fixing $M$, are also shown in the figure. This plot shows the largest differences occur at the boundaries and remain limited within the central part (note the different scale used for the differences plot). The orbital track pattern is also clearly visible here. It should be noted however that in this case there were no state vector differences between simulated orbits and the reduction orbits, since in the reduction run the a priori orbit was used, thus representing a best case scenario. In reality, there will always be state vector errors and one needs more flexibility in the orbit determination process, which can be obtained by estimating $M$. Therefore, when processing real Lunar Prospector observations, $M$ will not be fixed in the orbit determination. It is also expected that this reduces the orbital track patterns.
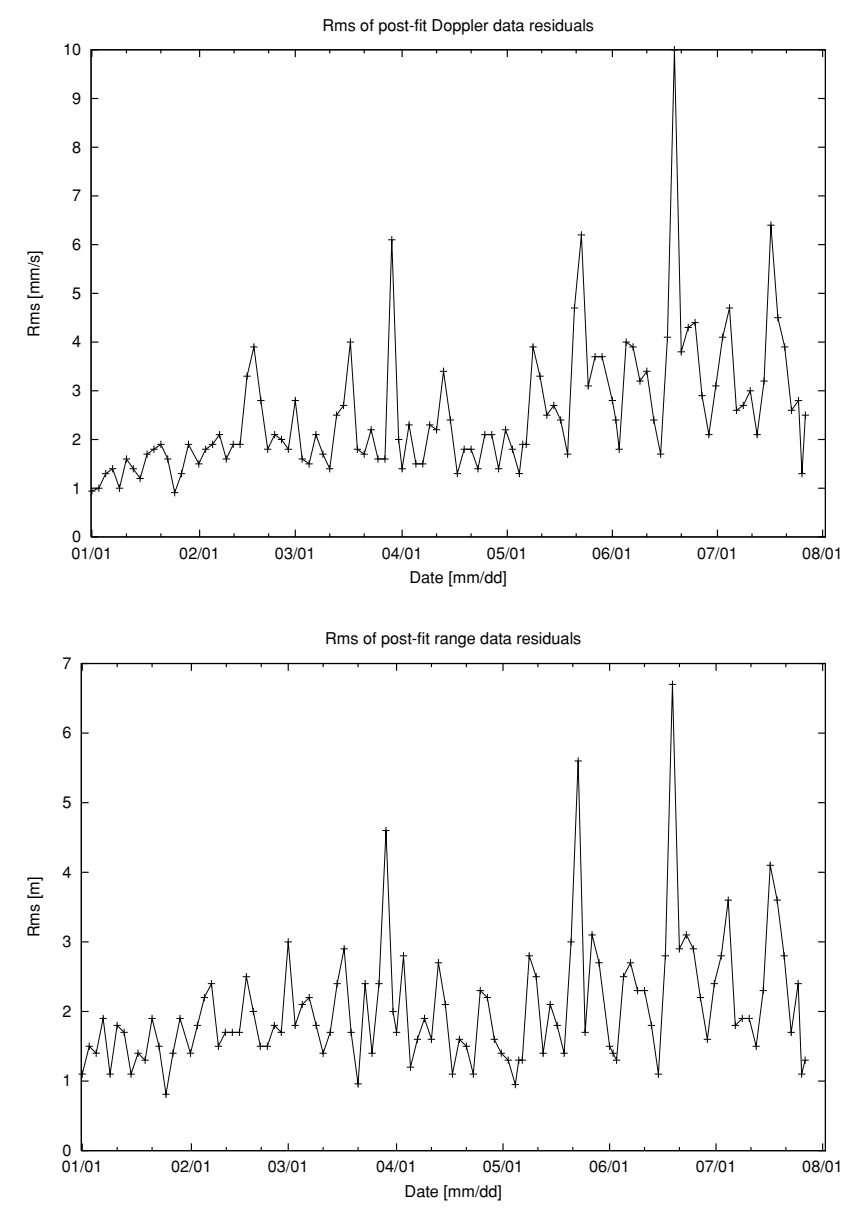

Fig. 3. Rms of fit for the Lunar Prospector Doppler and range residuals after orbit determination using two-day arcs for the months January until July, 1999.

Precise orbits need to be determined prior to the local recovery. This has been done by processing the entire Lunar Prospector data set for the last 7 months of the mission (January 1999 until July 31 1999), where its altitude was lowest at an average altitude of $30 \mathrm{~km}$ above the lunar surface. Using LP150Q up to degree 150 as the reference model and using a two-day arc length, the rms of residuals for Doppler data was typically better than $5 \mathrm{~mm} / \mathrm{s}$, and that for range data is at a level better than $3 \mathrm{~m}$, comparable to or better than (due to the use of a more recent a priori gravity field model) results published by Carranza et al. (1999). Results showing the rms of post-fit residuals per two-day arc for the entire extended mission for both Doppler and range data are shown in Fig. 3. Outliers appear to occur regularly in time and might be selenographically correlated. There is also a slight increase in rms of data fit towards the end of the entire Lunar Prospector mission. These orbits will deliver the initial state vector values for the short-arc orbit determination, from which the residuals will be used in the local recovery, cf. Fig. 1.

\section{Recovery Using a Pre-Lunar Prospector A Pri- ori Gravity Field Model}

To validate the method even further, a pre-Lunar Prospector gravity field model, namely GLGM-2 (Lemoine et al., 1997), will be used in the short-arc orbit determination. Ac- 


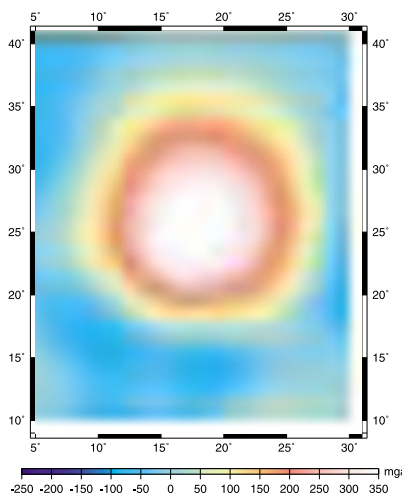

GLGM- $2, l=2-70$

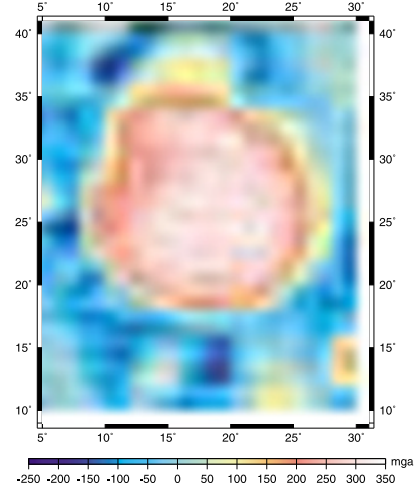

LP150Q, $l=2-150$

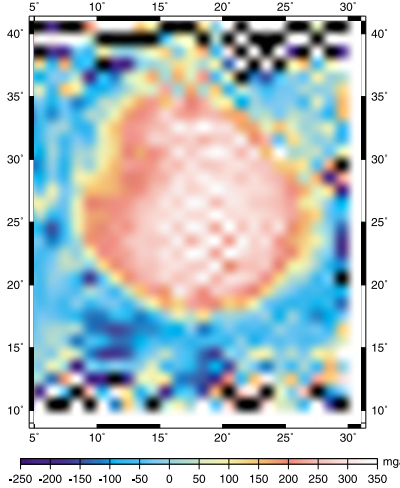

GLGM-2 + adjustment

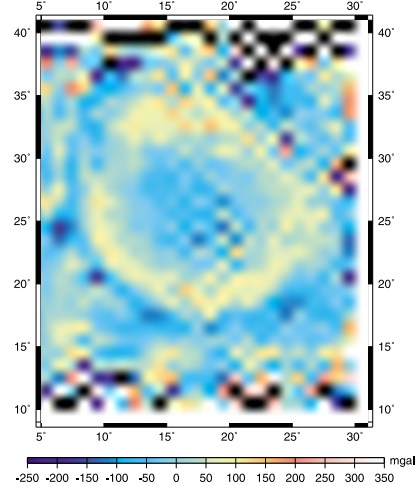

adjustment

Fig. 4. This plot shows anomalies according to GLGM-2 and LP150Q, the recovered anomalies when GLGM-2 is used in the short-arc orbit determination, and the adjustment itself, for the Mare Serenitatis area. These results use actual Lunar Prospector data. No regularisation was used to obtain the solution. Input anomalies using GLGM-2 have an rms of $198.05 \mathrm{mGal}$, those using LP150Q $213.5 \mathrm{mGal}$, for the central part $\left(\lambda=10^{\circ}-25^{\circ} \mathrm{E}, \phi=15^{\circ}-35^{\circ} \mathrm{N}\right)$. The new solution has an rms of 211.32 , and the adjustment itself of $61.53 \mathrm{mGal}$.

tual Lunar Prospector data will be used in this test so it is not a simulation where the recovered anomalies can be checked against a truth model. The advantage of using GLGM-2 is that this model is completely independent from Lunar Prospector data. The model will be used up to its maximum degree of 70 . Such a test can show that the recovery method is capable of extracting the high-frequency gravity field information present in the actual data. The results are shown in Figure 4. They have been obtained without regularisation, and the mean anomaly $M$ has not been fixed in the short-arc orbit determination.

Figure 4 shows that the new result for the anomalies of Mare Serenitatis using GLGM-2 as a priori model resembles the anomalies of the LP150Q model very well. Note that the new result was made entirely independent from the LP150Q model; this model is shown here for comparison since it is created using Lunar Prospector data. The improvement in resolution between the GLGM-2 model and the new result is clearly visible. In the adjustment itself a ring structure of anomalies of roughly the same size of the mascon can be seen, surrounded by negative adjustments. This same ring structure can also be seen when the differences in anomalies between those coming from LP150Q and those coming from GLGM-2 are investigated. It can clearly be seen that the solution at the boundary is less reliable.

The influence of estimating the mean anomaly $M$ or not has also been investigated. Differences for the results between estimating $M$ or not are profound and can be in the order of $30 \mathrm{mGal}$. If $M$ is kept fixed in the short-arc orbit determination, the adjustment has an rms for the inner part of $82.34 \mathrm{mGal}$, which is much larger than the $61.53 \mathrm{mGal}$ that is found when $M$ is not fixed. Not fixing $M$ reduces the chance of leakage of spurious signals into the adjustments.

In conclusion, the results show that the recovery method is indeed capable of extracting the high-frequency signal from the actual tracking data. Moreover, by using an older a priori model, the strength and information content of the Lunar Prospector data is made clearly visible, cf. Figure. 4.

\section{Results for Mare Serenitatis}

With the recovery method validated by different means, the final adjustment for the Mare Serenitatis area can be created. To show the area under study, Fig. 5 displays the topography of Mare Serenitatis, taken from a Digital Elevation Model created using the Clementine laser ranging instrument LIDAR (Smith et al., 1997). This figure shows that the areas near the margins of the mare are lower than the centre. East/west trends in the topography across the basin however show a less prominent central topographic high (Watters and Konopliv, 2001).

Data residuals from short-arc orbit determination from 7 months of Lunar Prospector tracking data are used. Per month there are roughly 52 tracks (or arcs) over the selected area, resulting in 52 normal matrices per month that need to be formed. The normal matrices are formed using LP150Q up to degree 150 as the reference gravity field model. All adjustments will be relative to this model. Few of these tracks were found to be unstable, leading to an unrealistically high Doppler data fit for that track (in the order of $10^{+3} \mathrm{~mm} / \mathrm{s}$ ). These outliers were discarded. All other normal matrices were included. The average Doppler data fit over the tracks was found to be $2.5 \mathrm{~mm} / \mathrm{s}$, with very few outliers of around $17.0 \mathrm{~mm} / \mathrm{s}$. Since range data are more scarce and noisier (Konopliv et al., 2001), only Doppler data residuals are used.

The new solution for Mare Serenitatis and the adjustment itself are shown in Fig. 6. Again, this solution was obtained without the use of a priori information. The new solution is very similar to the a priori anomaly field as was shown in Fig. 4. This can also be seen in the plot for the adjustment itself, since this is relatively flat. Nevertheless, an increase in resolution is visible, and the ring of negative anomalies surrounding the mascon appears to be more continuous, especially at the Southern border, which suggests that the highfrequency features have some physical reality. This can also be seen in the adjustment plot, where along the border of the mascon some anomaly adjustments larger than the average value surrounding them can be found. Considering the boundary effects present on such a solution, the ring feature might be better addressed using a larger recovery area. The 


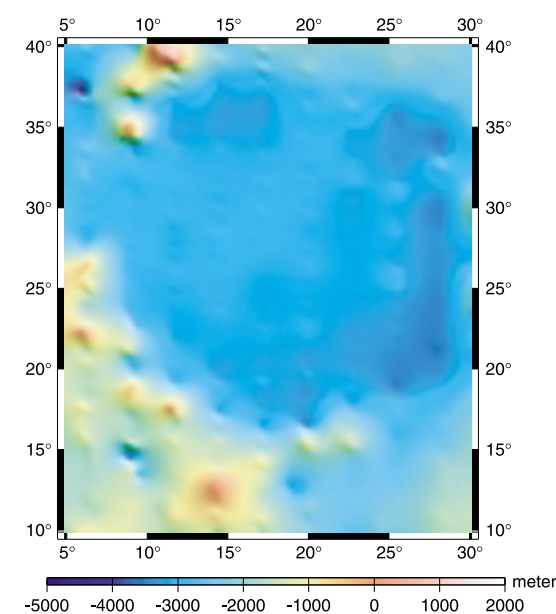

Fig. 5. Topography of Mare Serenitatis from the Clementine LIDAR instrument in a $0.25^{\circ} \times 0.25^{\circ}$ grid. Elevations are in meters above a reference spheroid with a radius of $1738 \mathrm{~km}$ and a flattening of $1 / 3234.93$, corresponding to the flattening of the selenoid.

adjustment itself does not show any clear, new concentration of anomalies, but it can be seen that inside the mascon, around the area $\lambda \approx 20^{\circ} \mathrm{E}, \phi \approx 25^{\circ} \mathrm{N}$, there is an increase in resolution. This is also visible when the adjustment itself is inspected, since the anomalies are larger there. The postfit Doppler residuals have also been investigated, showing a slight improvement in rms of fit of $0.13 \mathrm{~mm} / \mathrm{s}$ on an average level of $2.55 \mathrm{~mm} / \mathrm{s}$. This also shows that the adjustments are relatively flat and that the a priori model LP150Q already contains most of the gravity information present in the data. The remaining residuals might be due to either unmodelled ionospheric effects, or unmodelled effects of the spacecraft spin rate, see e.g. Konopliv et al. (2001). When compared to a similar solution as obtained by Sugano and Heki (2004), it is noticeable that in that solution a striped pattern is visible inside the mascon. Such a pattern can not be confirmed by either the global models or the local adjustment as presented here.

The results so far did not make use of a priori information. However, downward continuation of errors at satellite altitude leads to amplified errors in the solution, which then requires the use of a priori information in order to limit the effect of downward continuation. Simulations have shown that in case low-altitude data are used, a priori information is not a pre-requisite in order to obtain good solutions (Goossens, 2005; Goossens et al., 2005). Nevertheless, this has been investigated by adding an a priori covariance matrix for the anomalies, as was done by e.g. Barriot and Balmino (1992). This covariance matrix consists of a commission part, by including anomaly covariances coming from either the LP75G (Konopliv et al., 1998) covariance matrix (when LP150Q is used as a priori gravity field model) or from the GLGM-2 covariance matrix (when GLGM-2 is the a priori gravity field model), and an omission part, by including an isotropic and homogeneous covariance function which makes use of a Kaula rule for the Moon of $1.5 \cdot 10^{-4} / l^{2}$, e.g. Lemoine et al. (1997), where $l$ is the harmonic degree. This a priori covariance matrix thus describes the expected statistical behaviour of the anoma-
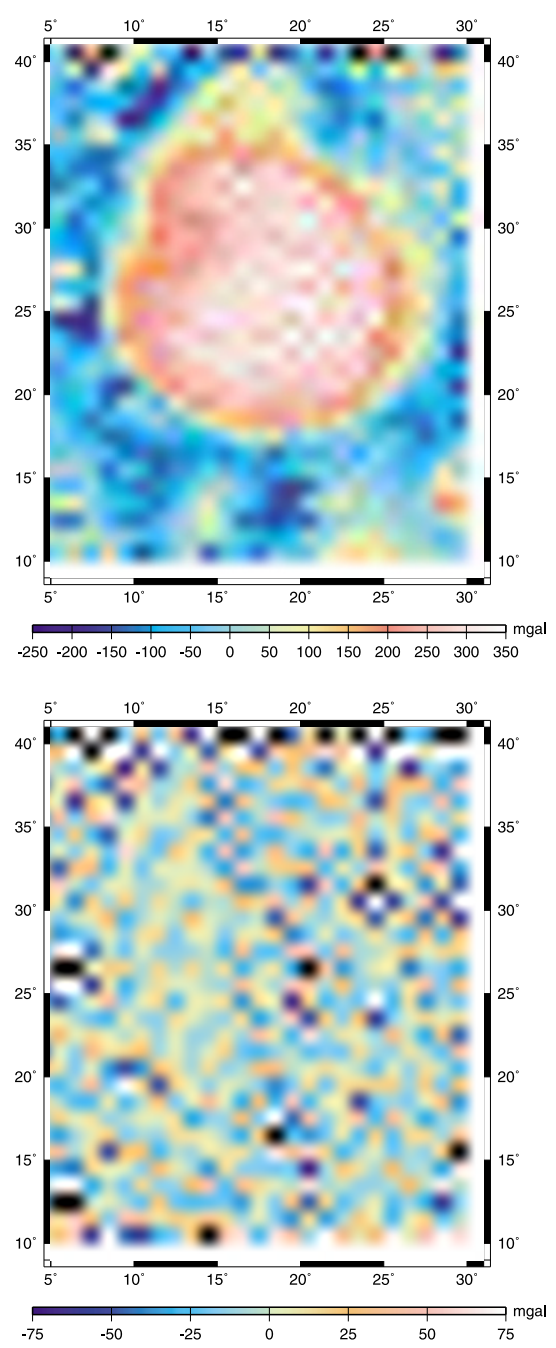

Fig. 6. New result for Mare Serenitatis and the adjustment itself, obtained from Lunar Prospector tracking data. The rms of the new solution for the central part $\left(\lambda=10^{\circ}-25^{\circ} \mathrm{E}, \phi=15^{\circ}-35^{\circ} \mathrm{N}\right)$ is $214.36 \mathrm{mGal}$; for the adjustment it is $25.87 \mathrm{mGal}$. Both values are for the central part $\left(\lambda=10^{\circ}-25^{\circ} \mathrm{E}, \phi=15^{\circ}-35^{\circ} \mathrm{N}\right)$.

lies at the surface. With the full a priori covariance matrix $\mathbf{C}_{\Delta g}$ thus constructed, the solution $\hat{x}_{\beta}$ can be computed from $\hat{x}_{\beta}=\left(\mathbf{A}^{T} \mathbf{W A}+\beta \mathbf{C}_{\Delta g}^{-1}\right)^{-1} \mathbf{A}^{T} \mathbf{W} \vec{y}$, where $\beta$ is the weight of the a priori information. The weight $\beta$ has been determined by minimising the differences in rms between solutions obtained using either LP150Q or GLGM-2 as a priori gravity field model. The smallest rms of differences will thus provide an optimal solution in the sense of the most consistent one when different a priori models are used. The results of this search are depicted in Fig. 7, where the rms of differences is plotted versus the regularisation parameter. This figure was created by searching for the parameter $\beta$ that would minimise the rms of differences, by first starting with values close to $\beta=1$ and then gradually changing the parameter until the minimum was found. This Figure shows that $\beta=10^{-6}$ produces the smallest rms of differences between the solutions of $22.67 \mathrm{mGal}$, versus an rms of differences of $29.25 \mathrm{mGal}$ when no a priori information is used. Both these differences are within the formal errors coming from either LP75G or GLGM-2 covariance, where 


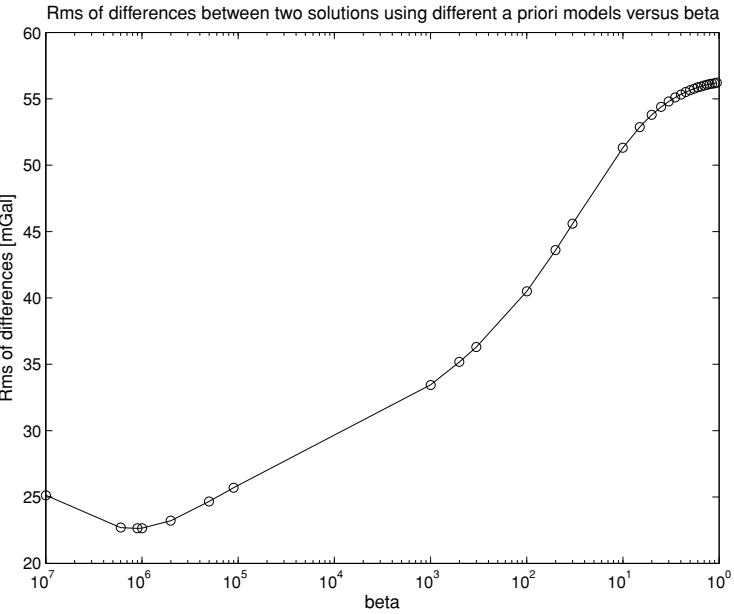

Fig. 7. Rms of differences between solutions using either LP150Q or GLGM-2 as a priori model, versus the regularisation parameter $\beta$.

it should be kept in mind that for the LP models a note is given that the real error in the selenopotential is probably a factor of two higher (Konopliv et al., 2001), due the negligence of systematic effects (Floberghagen, 2002). The differences between solutions using a priori information with $\beta=10^{-6}$ and using no a priori information (both using LP150Q as a priori gravity field model) are in the order of $10^{-4} \mathrm{mGal}$. These solutions are mostly determined by the data themselves, which is already seen from the low weight of the a priori information. The figure also shows that for $\beta=1$ the differences between both solutions are biggest. This is mostly because the solution using GLGM-2 as a priori model is severely damped when this value is used for $\beta$. This is much less the case for the solution using LP150Q as a priori model. This, and the small value of $\beta$ can indicate that further calibration of the a priori information might be required, but it should be kept in mind that the a priori information applied so far is based on information from global models which already required a priori information (in the form of a Kaula rule) themselves. Furthermore, it should also be kept in mind that overweighting the a priori information leads to dampening the amplitudes of the anomalies (Barriot and Balmino, 1994).

\section{Conclusions}

It has been demonstrated that the local recovery method can be applied to create high-resolution local gravity fields on the Moon. Processing the Lunar Prospector data in a precise orbit determination in order to create the residuals from which the anomalies are estimated, allows a comprehensive and detailed local gravity field determination. Results for Mare Serenitatis showed the possibility to increase the resolution of current global gravity field models. Without a priori information a good solution can be obtained. The weight of a priori information in the form of an full covariance matrix was determined by comparing two solutions using different a priori gravity field models. This weight was found to be very small, showing the solution mostly relies on the data themselves.
Acknowledgments. The authors would like to thank the following people: Dr. Alex Konopliv from JPL for providing the raw tracking data and the LP75G square root information filter, and Dr. Frank Lemoine and Dave Rowlands from NASA/GSFC for providing the GEODYN software, for providing the GLGM-2 covariance matrix, and for providing their preprocessing software to process ramped Doppler and range data. Part of this work was done when S. Goossens was on a short-term fellowship visiting NAOJ which was awarded by the Japan Society for the Promotion of Science (JSPS).

\section{References}

Barriot, J. P. and G. Balmino, estimation of local planetary gravity fields using line of sight gravity data and an integral operator, Icarus, 99(1), 202-224, 1992

Barriot, J. P. and G. Balmino, Analysis of the LOS gravity data set from cycle 4 of the Magellan Probe around Venus, Icarus, 112(1), 34-41, 1994.

Barriot, J.-P., G. Balmino, and N. Valès, Building reliable local models of the Venus gravity field from the Cycles 5 and 6 of the Magellan LOS gravity data, Geophysical Research Letters, 24(4), 477-480, 1997.

Carranza, E., A. Konopliv, and M. Ryne, Lunar Prospector Orbit Determination Uncertainties Using The High Resolution Lunar Gravity Field Models, in Advances in the Astronautical Sciences, AAS/AIAA Astrodynamics Specialist Conference, vol. 103, pp. 381-400, Girdwood, Alaska, AAS paper 99-325, 1999.

Floberghagen, R., Lunar Gravimetry, vol. 273 of Astrophysics and Space Science Library, Kluwer Academic Publishers, Dordrecht, The Netherlands, 2002.

Goossens, S., The Near Side-Regional Lunar Gravity Field Determination, Ph.D. dissertation, Delft University of Technology, 2005.

Goossens, S., P.N.A.M. Visser, and B.A.C. Ambrosius, A method to determine regional lunar gravity fields from earth-based satellite tracking data, Planetary and Space Science, 53, 1331-1340, 2005.

Heiskanen, W. A. and H. Moritz, Physical Geodesy, Institute of Physica Geodesy, Graz, Austria, Original edition by W. H. Freeman and Company, San Francisco, 1967, 1984.

Konopliv, A. S., A. B. Binder, L. L. Hood, A. B. Kucinkas, W. L. Sjogren, and J. G. Williams, Improved Gravity Field of the Moon from Lunar Prospector, Science, 281(5382), 1476-1480, 1998.

Konopliv, A. S., S. W. Asmar, E. Carranza, W. L. Sjogren, and D. N. Yuan, Recent gravity models as a result of the Lunar Prospector mission, Icarus, 150, 1-18, 2001.

Lemoine, F. G. R., D. E. Smith, M. T. Zuber, G. A. Neumann, and D. D. Rowlands , A 70th degree lunar gravity model (GLGM-2) from Clementine and other tracking data, Journal of Geophysical Research, 102(E7), 16,339-16,359, 1997.

Rowlands, D., J. A. Marshall, J. McCarthy, D. Moore, D. Pavlis, S. Rowton, S. Luthcke, and L. Tsaoussi, GEODYN II system description, Vols. 1-5, Contractor report, Hughes STX Corp., Greenbelt, MD, 1995.

Smith, D. E., M. T. Zuber, G. A. Neumann, and F. G. Lemoine, Topography of the Moon from the Clementine lidar, Journal of Geophysical Research, 102(E1), 1591-1611, 1997.

Sugano, T. and K. Heki, High resolution gravity anomaly map from the Lunar Prospector line-of-sight acceleration data, Earth Planets Space, 56, 81-86, 2004.

Thornton, C. L. and J. S. Border, Radiometric Tracking Techniques for Deep-Space Navigation, Monograph 1, Deep-Space Communications And Navigation Series, Jet Propulsion Laboratory, October 2000, JPL Publication 00-11, 2000.

Vonbun, F. O., W. D. Kahn, W. T. Wells, and T. D. Conrad, Determination of $5^{\circ} \times 5^{\circ}$ gravity anomalies using satellite-to-satellite tracking between ATS-6 and Apollo, Geophys. J. R. astr. Soc., 61, 645-657, 1980.

Watters, T. R. and A. S. Konopliv, The topography and gravity of Mare Serenitatis: implications for subsidence of the mare surface, Planetary and Space Science, 49, 743-748, 2001.

S. Goossens (e-mail: sander@miz.nao.ac.jp), P. N. A. M. Visser, K Heki, and B. A. C. Ambrosius 